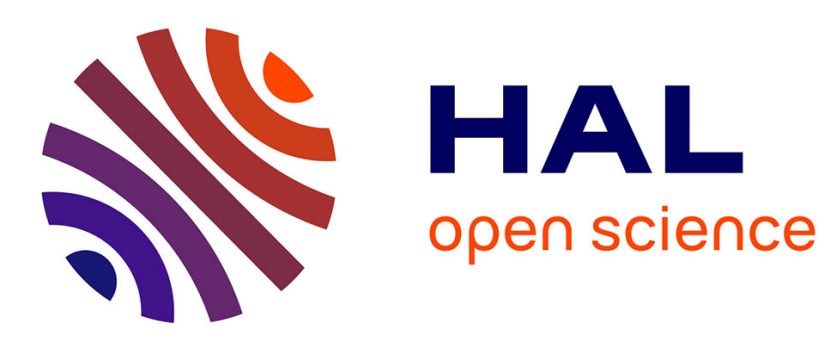

\title{
High-Order Numerical Methods for KEEN Wave Vlasov-Poisson Simulations
}

Michel Mehrenberger, Nicolas Crouseilles, Eric Sonnendrücker, Bedros Afeyan

\section{To cite this version:}

Michel Mehrenberger, Nicolas Crouseilles, Eric Sonnendrücker, Bedros Afeyan. High-Order Numerical Methods for KEEN Wave Vlasov-Poisson Simulations. PPPS, Jun 2013, San Francisco, United States. 10.1109/PLASMA.2013.6634958 . hal-01298979

\section{HAL Id: hal-01298979 \\ https://hal.science/hal-01298979}

Submitted on 6 Apr 2016

HAL is a multi-disciplinary open access archive for the deposit and dissemination of scientific research documents, whether they are published or not. The documents may come from teaching and research institutions in France or abroad, or from public or private research centers.
L'archive ouverte pluridisciplinaire $\mathbf{H A L}$, est destinée au dépôt et à la diffusion de documents scientifiques de niveau recherche, publiés ou non, émanant des établissements d'enseignement et de recherche français ou étrangers, des laboratoires publics ou privés. 


\section{High-Order Numerical Methods for KEEN Wave Vlasov-Poisson \\ Simulations}

I9MA Institut de Recherche
Mathématique Avancée

\author{
Michel Mehrenberger ${ }^{a}$ Nicolas Crouseilles ${ }^{b}$ Eric Sonnendrücker ${ }^{c}$ \\ Bedros Afeyan ${ }^{d}$
}

${ }^{a}$ IRMA, Université de Strasbourg, France ${ }^{b}$ INRIA-Rennes, France ${ }^{c}$ Max-Planck-Institut für Plasmaphysik, Garching, Germany ${ }^{d}$ Polymath Research, Pleasanton, USA

mehrenbedmath.unistra.fr, crouseil@inria.fr, sonnendipp.mpg.de, bafeyanegmail.com

\section{Abstract}

KEEN waves [1] provided a challenging test case for Vlasov Poisson numerical solvers since they involve highly non stationary, multiple-harmonic self-organized highly non stationary, multiple-harmonic self-organized
kinetic states. They require high resolution in the phase space region around the phase velocity of the drive wave. Different interpolation strategies are discussed and compared to classical cubic splines.

\section{KEEN wave test case}

We solve Vlasov-Poisson equation

$\partial_{t} f+v \partial_{x} f+\left(E-E_{\text {app }}\right) \partial_{v} f=0, \partial_{x} E=\int_{\mathbb{R}} f d v-1$,

where $E_{\text {app }}(t, x)$ is of the form

$$
E_{\text {app }}(t, x)=E_{\max } k a(t) \sin (k x-\omega t),
$$

where

$$
\begin{aligned}
& a(t)=\frac{0.5\left(\tanh \left(\frac{t-t_{L}}{t_{w L}}\right)-\tanh \left(\frac{t-t_{R}}{t_{w R}}\right)\right)-\epsilon}{1-\epsilon}, \\
& \epsilon=0.5\left(\tanh \left(\frac{t_{0}-t_{L}}{t_{w L}}\right)-\tanh \left(\frac{t_{0}-t_{R}}{t_{w R}}\right)\right)
\end{aligned}
$$

is the amplitude, $t_{0}=0, t_{L}=69, t_{R}=307, t_{w L}=t_{w R}=$ $20, k=0.26, \omega=0.37$ and $E_{\max }=0.2$. The initial condition is

$$
f_{0}(x, v)=\frac{1}{\sqrt{2 \pi}} \exp \left(-\frac{v^{2}}{2}\right), \quad(x, v) \in[0,2 \pi / k] \times[-6,6] .
$$

\section{Implementation on uniform mesh}

Strang (or higher order) splitting leads to

$$
\partial_{t} f+v \partial_{x} f=0, \partial_{t} f+\left(E-E_{\text {app }}\right) \partial_{v} f=0,
$$

i.e. $N \in\left\{N_{x}, N_{v}\right\} 1 D$ constant advection equations

$$
\partial_{t} u+c \partial_{x} u=0, u=u(t, x)
$$

$t_{n+1} \quad$. $u_{i}^{n+1} \simeq u\left(t_{n+1}, x_{i}\right)=u\left(t_{n}, x_{i}-c \Delta t\right)$.

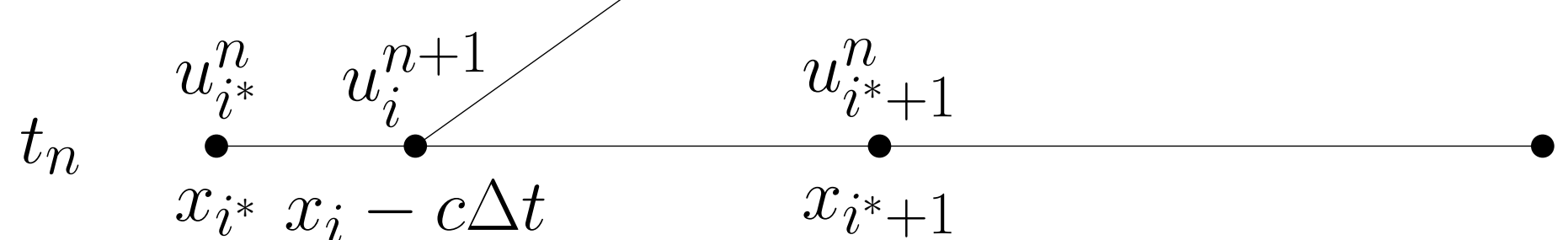

We can write $u^{n+1}=A u^{n}$ with circulant matrix

$$
A:=\left(\begin{array}{ccccc}
a_{0} & a_{1} & \ldots & \ldots & a_{N-1} \\
a_{N-1} & a_{0} & a_{1} & \ldots & a_{N-2} \\
\ddots & \ddots & \ddots & \ddots & \ddots \\
\ddots & \ddots & \ddots & \ddots & \ddots \\
a_{1} & \ldots & \ldots & a_{N-1} & a_{0}
\end{array}\right)
$$

- Use of FFT for efficient matrix/vector product

- Same form for different interpolations:

- LAG(2d+1): symmetric Lagrange of order $p=2 d+1$

-SPL(p): $B$-splines of order $p$

- SPL3 corresponds to classical cubic splines

- Almost same complexity independently of $k$

\section{Acceleration on GPU}

Use of existing GPU kernels

- Transposition

- cufft for complex FFT

- Adaption of ScalarProd for charge density:

$$
\sum f_{i}=\langle f, 1\rangle
$$

Kernel for computing $A$ :

- analytical formula for $\mathrm{LAG}-(2 \mathrm{~d}+1)$

- switch complexity from $O(N d)$ to $O\left(N d^{2}\right)$

Dealing with single precision issues:

- $\delta$-f method
Modification of $v$-advection

$$
\delta f\left(x, v-\Delta t E^{n}(x)\right)+f_{\mathrm{eq}}\left(v-\Delta t E^{n}(x)\right)-f_{\mathrm{eq}}(v)
$$

- Zero mean condition for charge density $\rho=\int f d v$

$$
\tilde{\rho}_{k}=\rho_{k}-M, M=\frac{1}{N} \sum_{k=0}^{N-1} \rho_{k},
$$

instead of $\tilde{\rho}_{k}=\rho_{k}-1$, whose RHS is only approximatively of zero mean.

4. Cubic Hermite formulation on uniform mesh

Classical cubic splines method is reinterpreted as cubic Hermite interpolation with particular choice of derivative reconstruction leading to a tridiagonal system for tive recons
$u_{j^{+}}^{\prime}=u_{j^{-}}^{\prime}$

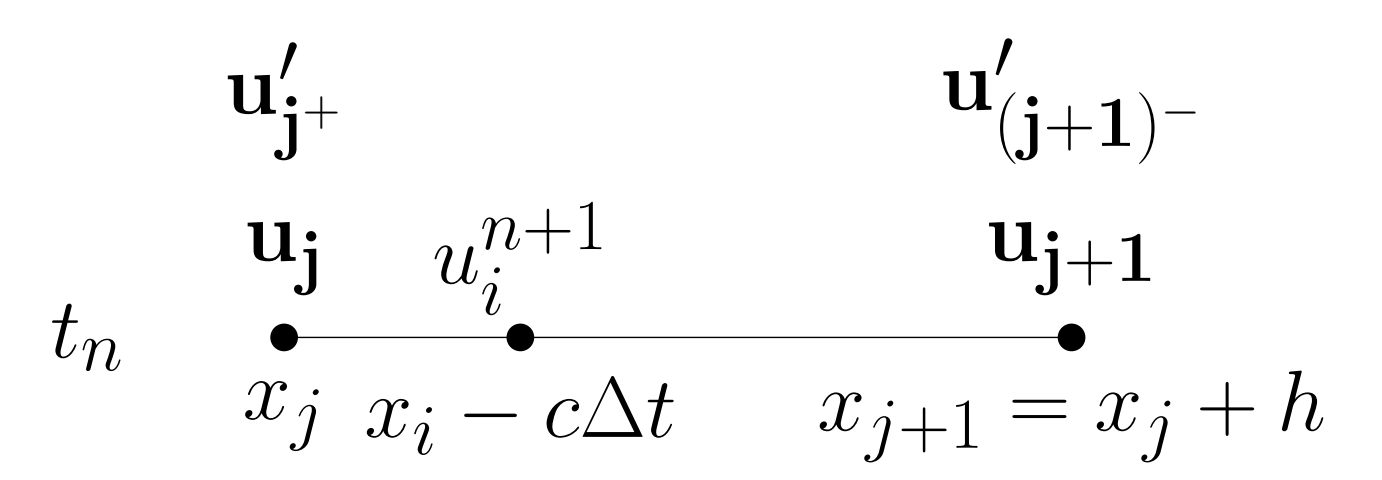

$\Rightarrow$ In this framework, we can choose other reconstructions for the derivatives

$\mathbf{F D}(\mathbf{p})$ : compact finite difference of order $p$

$u_{j^{+}}^{\prime}, u_{(j+1)^{-}}^{\prime}$ : formula with stencil $j-\left\lfloor\frac{p}{2}\right\rfloor, j+\left\lfloor\frac{p+1}{2}\right\rfloor$

- The order $p$ is for point derivatives not for interpolation which remains third order

- Formulae are explicit, which permits easy change of parameter $p$ in the code

- Interpolation becomes local and remains third order

$\rightarrow$ generalizations not prohibitive w.r.t cost

- For $p$ even, we get a $C^{1}$ reconstruction with $u_{j^{+}}^{\prime}=u_{j^{-}}^{\prime}$

- For $p$ odd, upwinding effect; better for small $\Delta t$

$\rightarrow$ no dispersion effect as for $p$ even or SPL3

- FD3=LAG3

- $\mathrm{FD}(2 \mathrm{~d}+1) \simeq \mathrm{LAG}(2 \mathrm{~d}+1), d \geq 2$

- equality for limit $\Delta t \rightarrow 0$

- schemes remain different, as $\mathrm{FD}(2 \mathrm{~d}+1)$ is third order - $\mathrm{FD} 6 \simeq \mathrm{SPL} 3$

\section{Description of a simple non uniform mesh}

Higher resolution needed for velocity around $\omega / k$

$\Rightarrow$ We have to adapt the methods to deal with a uniform mesh with a refined zone

Mesh spacing on coarse/fine grids are

$$
\Delta v_{\text {coarse }}=\frac{v_{\max }-v_{\min }}{N_{\text {coarse }}}, \Delta v_{\text {fine }}=\frac{v_{\max }-v_{\min }}{N_{\text {fine }}}
$$

and $N_{\text {fine }}$ is an integer multiple of $N_{\text {coarse. }}$.

The refined zone is chosen with $0 \leq i_{1}<i_{2} \leq N_{\text {coase }}$ and the total number of cells is

$$
\begin{aligned}
& N=i_{1}+N_{f}+N_{\text {coarse }}-i_{2}, N_{f}=\frac{N_{\text {fine }}}{N_{\text {coarse }}}\left(i_{2}-i_{1}\right) \\
& N_{\text {coarse }}-i_{2} \\
& \text { • } \bullet \cdot \\
& \text { • • • }
\end{aligned}
$$

6. Hermite formulation on such mesh

Classical non uniform cubic splines can be used and are again reinterpreted as cubic Hermite interpolation with a particular choice of derivative reconstructions with a particular choice of derivative reconst

- Works for arbitrary non uniform mesh (not only uniform refined mesh)

For using the specificity of this uniform refined mesh, we can use a two-grid reconstruction for the derivatives: Two-grid cubic splines:

- Compute derivatives on coarse grid points to get it at points
- Compute it on fine grid points

$$
v_{j}, j \in\left\{i_{1}, \ldots, i_{1}+N_{f}\right\},
$$

using boundary conditions at points $v_{i_{1}}, v_{i_{1}+N_{f}}$

As in the case of uniform grid, we can adapt the reconstruction of derivatives in the $\mathrm{FD}(\mathrm{p})$ case.

Two-grid FD(p):

- Compute derivatives using $F D$ formula on coarse grid

- Compute function values on some boundary fine grid points in $\left[v_{0}, v_{i_{1}}\right] \cup\left[v_{i_{1}+N_{f}}, v_{N}\right]$, that are needed for next step, using interpolation on coarse grid

- Compute derivatives using $F D$ formula on fine grid

7. Conservative version

Previous version has to be changed on non uniform grids in order to be mass conservative.

- Unknowns are $u_{j+1 / 2}=\frac{1}{v_{j+1}-v_{j}} \int_{v_{j}}^{v_{j+1}} u(v) d v$

- Use of previous Hermite interpolation on primitive data

$$
U\left(v_{j}\right)=\int_{v_{0}}^{v_{j}} u(y) d y, v_{j}, j=0, \ldots, N
$$

- Choose adhoc integration constant for dealing with a primitive that is also periodic

\section{Numerical results}

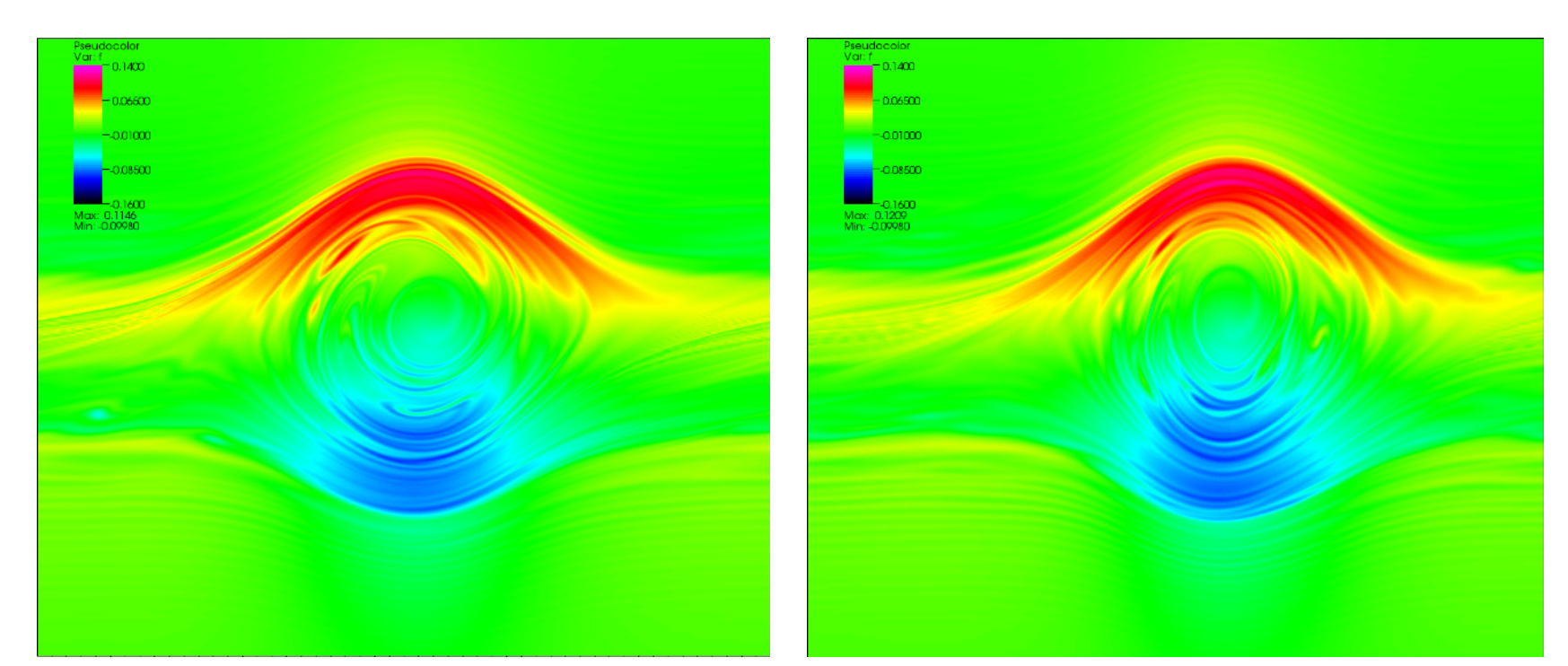

Figure 1: $f(1000, x, v)-f_{0}(x, v), \Delta t=0.05$. Comparison of SPL3, with $N_{x}=N_{v}=4096$ on CPU (left) and LAG17 $N_{x}=N_{v}=2048$ on GPU double precision (right).
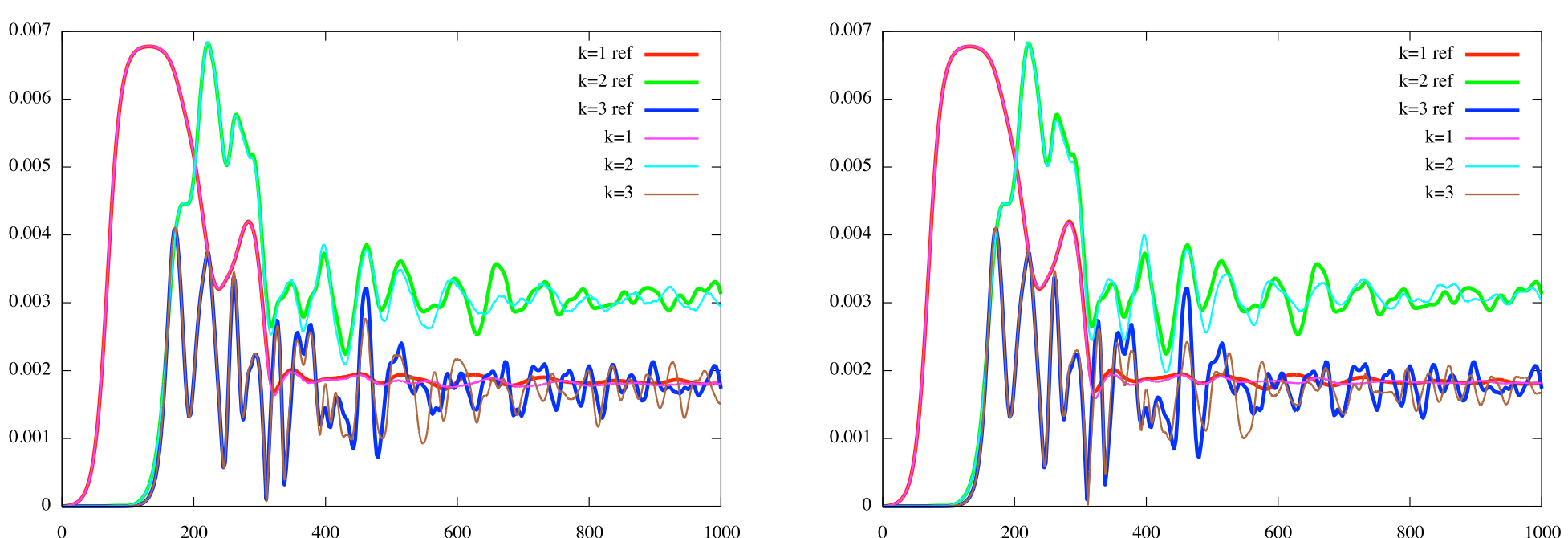

Figure 2: Absolute values of the first three Fourie modes of $\rho$ vs time. Reference solution with LAG17 $N_{x}=$ $N_{v}=2048$ on GPU double precision (red, green and blue) compared to solution on uniform mesh in space (LAG17, $N_{x}=256$ ) and uniform refined mesh in velocity with $N_{v}=374$ ( $\left.N_{\text {coarse }}=64, N_{\text {fine }}=2048, i_{1}=34, i_{2}=44\right)$ (left): conservative non uniform cubic splines (right): conservative two-grid FD5

\section{Conclusion}

- Efficient GPU $1 D \times 1 D$ uniform Vlasov-Poisson solver - double precision: $2048 \times 2048,30$ Gflops - single precision: $4096 \times 4096,100$ Gflops

- Non uniform $1 D \times 1 D$ Vlasov-Poisson solver

- number of points are reduced

- encouraging results for future $2 D \times 2 D$ simulations

\section{References}

[1] B. Afeyan, K. Won, V. Sachenko et al., Kinetic Elec trostatic Electron Nonlinear (KEEN) waves and their interactions driven by the ponderomotive force of crossing laser beams, IFSA Proceedings 2003 and ArXiv:1210.8105

[2] M. Mehrenberger, C. Steiner, L. Marradi, N. Crouseilles, E. Sonnendrücker, B. Afeyan, Vlasov on GPU, submitted to ESAIM Proceedings, 2013. 